\title{
Project Design and Implementation of Face Recognition, Fever Detection, and Attendance Record Based on Sensing Technology
}

\author{
Min-Chuan Huang, ${ }^{1}$ I-Ping Chen, ${ }^{2}$ Hsiang-Lin Huang, ${ }^{3}$ \\ Shih-Fu Sung, ${ }^{4}$ and Ai-Guo Wang ${ }^{1 *}$ \\ ${ }^{1}$ Guangdong University of Petrochemical Technology, \\ No. 139, Guandu 2nd Road, Maoming City, Guangdong 525000, China \\ ${ }^{2}$ Central Taiwan University of Science and Technology, \\ No. 666 Linzi Road, Beitun District, Taichung 406053, Taiwan \\ ${ }^{3}$ School of Management, Ming Chuan University, No. 250, Sec. 5, Zhongshan North Road, Taipei 10012, Taiwan \\ ${ }^{4}$ Department of Information Management, I-Shou University, \\ No. 1, Sec. 1, Syuecheng Rd., Dashu District, Kaohsiung 83012, Taiwan
}

(Received November 28, 2020; accepted April 6, 2021)

Keywords: face recognition, attendance software, Django, MySQL, sensing technology

We applied sensing principles using sensing technology, redeveloped efficient software using its standardized methods, and expanded the use of networks. The sensing principle and sensing technology can be applied to research and analysis in business processes such as facial recognition, body temperature testing, and automated attendance recording of web technology. On the basis of the results of market demand analysis, we successfully proposed a system to simplify the design, demonstrating the various functions of the attendance recognition software based on web technology. The new system avoids the deficiencies of the old system, making it more innovative. We used software such as HTML, CSS, JS, Django, MySQL, and WeChat programs among multiple frameworks and developments for our system, and used MySQL to store employee facial information, basic personal information, attendance records, personal temperature test results, and health records.

\section{Introduction}

With the rapid development of information technology, technologies in various fields such as computer hardware and software have improved. Artificial intelligence (AI) and automation technology, in particular, have been pushed toward the directions of medicine, health, and life in space technology, working to gradually increase human life expectancy and improve the quality of life. At the time of writing, there have been more than 110 million confirmed cases and 2730000 deaths caused by COVID-19 globally. The total number of confirmed cases in the United States has exceeded 30570000 and the number of deaths is 555000.(1) At present, COVID-19 is still spreading all over the world, not only disrupting people's lives but also raising the awareness of disease prevention. As COVID-19 spreads and mutates rapidly, countries

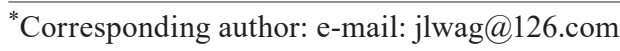

https://doi.org/10.18494/SAM.2021.3203 
around the world have gradually increased preventive measures. The international economic and social order urgently needs to be restructured and restored. To maintain our livelihoods, we have to cautiously return to the workplace while living with the threat of COVID-19 and practicing self-protection and social distancing.

The teaching objective is to guide college graduates to innovate and design useful articles for life. ${ }^{(2,3)}$ During the pandemic, group discussions have been conducted through video conferencing, dividing experiments, mastering group progress, letting students self-integrate their knowledge, and verifying their learning experience and ideas from the past four years, so they can apply what they have learned. ${ }^{(4)}$ The principles and technology of sensing are applied to the research and analysis of business processes such as facial recognition, body temperature testing, and automated attendance recording using web technology. $(5,6)$

The purpose of this study is to establish a fever detection file database for returning workers, ${ }^{(7)}$ and use face recognition system technology to identify them and give them a green or red health code to indicate whether their current health allows them to enter the workplace. ${ }^{(8)}$ At the same time, a database for an attendance system is established, ${ }^{(9)}$ which can effectively monitor and control the current situation of disease prevention and contact time series, track the timeline and condition of patients with a fever, and establish a personal and group database. This is to achieve effective disease prevention and organizational care, and to improve the virtuous cycle of interpersonal cohesion and centripetal force. ${ }^{(10)}$

The first step is to screen applications and existing software packages connected to fever detection equipment and database planning. Starting from the standardized method of software system development for engineering courses, the goal is to use Internet mobile phones for faceto-face consultation. Mobile health applications (apps) can be used as part of telehealth to monitor patient-reported outcomes and enhance patient-provider communication. ${ }^{(11)}$

In recent years, international corporations have been looking for new and effective methods for monitoring employees' attendance and clocking in, hoping to move towards the current needs of companies and enterprises, and overcome the defects of the attendance mechanisms of magnetic cards and fingerprint technology. Just as license plate recognition and payment systems have been widely used by enterprises, the AI field, which is gradually adopting face recognition technology, is commonly used in the employee clock-in recognition mechanism and has gradually entered use in corporate employee attendance system databases. Face recognition is an image processing technology to detect human face features, ${ }^{(12)}$ which extracts 128 biological characteristics of human face information. On the basis of an image library or on-thespot photography, element points of the facial contour structure can be quantified, and then the gender, identity, professional title, and job-related authority of the face can be identified through quantitative comparison and analysis.

The design and implementation of a system for facial recognition, fever detection, and attendance recording based on web technology can meet the requirements of corporate human resource management systems. This system can also provide information for enterprise attendance management and the scientific management of employee health, ${ }^{(13)}$ especially in the case of an emergency or for travellers. Cloud-based solutions have open challenges of interoperability and integration, higher challenges for security and privacy, and may lack $24 / 7$ 
support for the high availability of health history. Existing portable systems store limited health information for only a specific hospital and do not support mobility of patients across different hospitals. In this paper, we propose a next-generation portable Smart Health Record Management system with secure Near Field Communication (NFC). It will have many advantages, such as early fever warning, convenient health tracking and retrieval of employees, data security, reliable attendance data, a large storage capacity for data, low management cost, and the long service life of conventional equipment. Such a system will be essential to personal health and needs to be widely promoted.

\section{Methods}

Facial recognition is an interdisciplinary research of pattern recognition and computer vision. It originated in the 1960s. At that time, face recognition technology was mainly based on the research of facial contours. In recent decades, recognition technology has analyzed the facial features and contours of each person. A computer can easily distinguish even small differences among people, helping to distinguish them. Presently, face recognition technology is widely used in customs clearance detection systems when entering and leaving a country. Compared with fingerprint and iris recognition technology, facial recognition has similarities but also exceptional characteristics. These recognition technologies are similar in that they can identify each person's unique identity. Although irises and fingerprints can also be used to extract features, they may be damaged and changed as a result of an accident or surgery, whereas facial recognition features will not change with time or the environment. ${ }^{(14)}$

At present, many research institutions and overseas universities, including MIT, CMU Robotics Institute, Cornell University, and Berkeley University, have teams that have made significant contributions in the field of facial recognition. Domestic institutions studying facial recognition are the Chinese Academy of Sciences, Tsinghua University, Harbin University of Technology, and Nanjing University of Technology, along with other long-term research teams.

There are many kinds of attendance monitoring systems, such as those based on fingerprint recognition, ${ }^{(15)}$ iris recognition, ${ }^{(16,17)}$ and facial recognition. ${ }^{(18)}$ Old attendance monitoring systems have a barcode punch card or a magnetic card recorded in a database and were designed for convenience to replace the use of notes and coins. They have been widely used in various markets because the cost of the cards is low and the recognition accuracy is high. However, they can be lost, damaged, or stolen. The latest technology has been implemented in employee identification systems, inventories, security personnel displays, and RFID items related to patrol tracking records, disaster simulations, and impact factor analyses. In the future, 5G or B5G technology will be able to detect abnormal behaviors of employees due to physical discomfort or unintentional negligence, so disasters can be prevented in time. Recently, face recognition technology has been gradually introduced into large supermarkets for digital currency payment at check-out counters. ${ }^{(19)}$ At present, it is used for convenient cashflow trading, logistics shelf management, accurate customer group marketing, and customer loyalty management. It is expected that face recognition technology will move toward regional strategic operations and analyses in the future. 


\subsection{System analysis and system design}

By using the system architecture, the preliminary functional requirements of the system are satisfied. Details are shown in Table 1.

Table 1

Software selection analysis and application concept.

\begin{tabular}{ccc}
\hline \multirow{2}{*}{ Project } & \multicolumn{2}{c}{ Main content } \\
\cline { 2 - 3 } & $\begin{array}{c}\text { Software selection analysis } \\
\text { A very popular programming language for beginners. Simple, } \\
\text { easy to learn, and highly readable. Fits the thinking mode of our } \\
\text { life. Often used in many fields, such as artificial intelligence and } \\
\text { machine learning. Compatible with other languages, such as network } \\
\text { programming, the web, and GUIs. }\end{array}$ & $\begin{array}{c}\text { Python programming } \\
\text { language is simple, easy to } \\
\text { learn and maintain, and has a } \\
\text { powerful database. }\end{array}$ \\
\hline
\end{tabular}
A web framework written in Python. Adopts the classic MVC mode and is a three-tier structure. $\mathrm{M}$ is the data model; $\mathrm{T}$ is the view, which is used to write front-end page code; $\mathrm{V}$ is the controller used to write

Django functional logic code. Advantages are a perfect URL routing controller, simple configuration, easy to understand, high degree of freedom, and safe and reliable characteristics.

A structured relational database with a small volume and fast

With an automatic background management interface, form processing, and template system, it can achieve a decoupling effect.

A structured relational database with a small volume and fast
MySQL
command execution. Free to use, reducing user cost. Simple database,
easy to learn, and easy to use. Supports multiple languages and can run
on multiple systems. Open code that can be modified.

MySQL database runs quickly, is low cost, easy to use, portable, open source, and has good maintenance.

HTML language does not need to be resolved

HTML

The code used to design web pages. Has the basic structure for web page layout and is a necessary technology for making web pages.

by a specific server and is recognized and parsed by most browsers.

\begin{tabular}{c} 
CSS $\begin{array}{c}\text { Recognized in various browsers, independent of the operating system, } \\
\text { and can be compatible with editing on various platforms. Can be } \\
\text { referenced in many places without the need to write redundant code } \\
\text { and is highly flexible. }\end{array}$ \\
JavaScript $\begin{array}{c}\text { HTML can be used to build the foundation of a website, CSS can be } \\
\text { used to decorate web pages, and JavaScript can be used to interactively } \\
\text { present dynamic effects. }\end{array}$ \\
\hline
\end{tabular}

The function of CSS is to decorate a website in any style.

\section{HTML, CSS, and JavaScript} are the perfect combination for building web pages. The WeChat applet can be WeChat three parts: view layer, logic layer, and system layer. The WeChat applet is convenient, fast, and occupies little capacity used without downloading,

forming a community ecosystem.

Face Performed with Python's facial recognition library, which uses Dlib's recognition deep learning face recognition technology. Can detect facial images and support multiuser facial recognition at the same time.

Dlib is open source and free for all to use.

Can detect multiple people at the same time in crowded places. High passage efficiency and a temperature-measuring time of less than 1

Intelligent human body temperature monitoring system $\mathrm{s}$, which will not cause congestion. Can avoid contact between staff and suspected patients and reduce the risk of staff infection. High accuracy rate and low risk of false or missed detection. Wireless remote data transmission, providing real-time display of data and information transparency. Equipped with a large data display screen, so everyone can participate in supervision. Data can be stored flexibly, and historical curves can be analyzed at any time to achieve big data monitoring and management. 


\subsection{System feasibility analysis}

System feasibility analysis is divided into the following four analyses: technical feasibility analysis, economic feasibility analysis, operational feasibility analysis, and business process analysis

\subsubsection{Technical feasibility analysis}

The system architecture mainly adopts technology from Python, the Django framework, and the WeChat applet. Python is a simple and easy-to-use programming language with high developmental efficiency. It is also an open-source project. The Django framework has perfect functions, complete elements, and a large collection of commonly used tools and modules, making it suitable for the rapid development of application-oriented websites and saving development time. The WeChat applet is a WeChat product, which is very user friendly to developers and does not incur an extra cost; developers can simply register and begin developing.

\subsubsection{Economic feasibility analysis}

The sensing image of the camera is also displayed on the computer and TV screen for checking. The interface of the system is clean and smooth, and the control is simple, without a lot of complex operations. Intelligent human body temperature monitoring and attendance monitoring should be processed online, so that there is no need for large machinery or equipment.

\subsubsection{Operational feasibility analysis}

The face recognition attendance system does not need to have any third-party software installed; it only needs the client's computer with a web browser. The system is very straightforward and convenient for employees. Employees only need to carry out facial recognition using the system and then click to log in to check their daily temperature and attendance reports with the program. The WeChat applet has already been integrated into people's daily lives, and employees can view their records anytime and anywhere, helping them easily submit a leave application. It also provides a background system with simple operations and a clear interface for administrators, which greatly aids their management of employees' attendance information. Therefore, the system is feasible for operation.

\subsubsection{Business process analysis}

There are super administrators in the system, who have all the rights of the system. A new employee registers information via the administrator. The administrator enters the employee's facial and basic personal information into the system. The face recognition technology will identify whether the face is ambiguous and request that the user re-enters the information if 
needed. When successful, the job identification number and initial password are assigned to the employee so the employee can check their body temperature, fever status, and clock in and out times. Facial recognition is carried out by computer cameras, and a voice confirms the clock in as successful. The data is also transmitted to a database through the server to form attendance reports. Employees can check whether they clocked in successfully through the applet. The system calculates the statistics of the attendance records of employees monthly. The administrator can view the monthly attendance statistical table of each employee and a statistical pie chart of the total attendance rate of the whole company. Company employees can open their mobile WeChat app, search for the applet, click to log in to obtain authorization information, and enter the account number and password in the login page to view the day's attendance information and absence record.

\section{Results}

First, the overall design of the whole system is carried out in a general direction, and the overall architecture of the system is designed to provide the possibility of further detailed designs.

\subsection{Overall design system}

The description of the overall design of the system is divided into four parts: system logic architecture design, basic face information management, attendance statistics management, and system database design.

\subsubsection{System logic architecture design}

The face recognition attendance system is a comprehensive application based on a browser, sensor, and server. When the functional logic code on the server side completes the database, the data is returned to the client. The client does not need to know the implementation process, reducing the burden on the client. The browser and server $(B / S)$ structure of the system is divided into three layers: the presentation layer, logic layer, and data layer. The task of the presentation layer is to return the results page and data to the client, and it can directly report the interaction between the client and the server. The $\mathrm{B} / \mathrm{S}$ architecture of the system is shown in Fig. 1.

\subsubsection{Basic face information management}

The functional structure of the basic face information management, as shown in Fig. 2, includes five functional modules for attendance management: a face information module, an employee information module, a fever detection information module, a clock-in time management function module, and a system information management function module. Figure 2 shows a flow chart of the functional structure of basic face information management. 


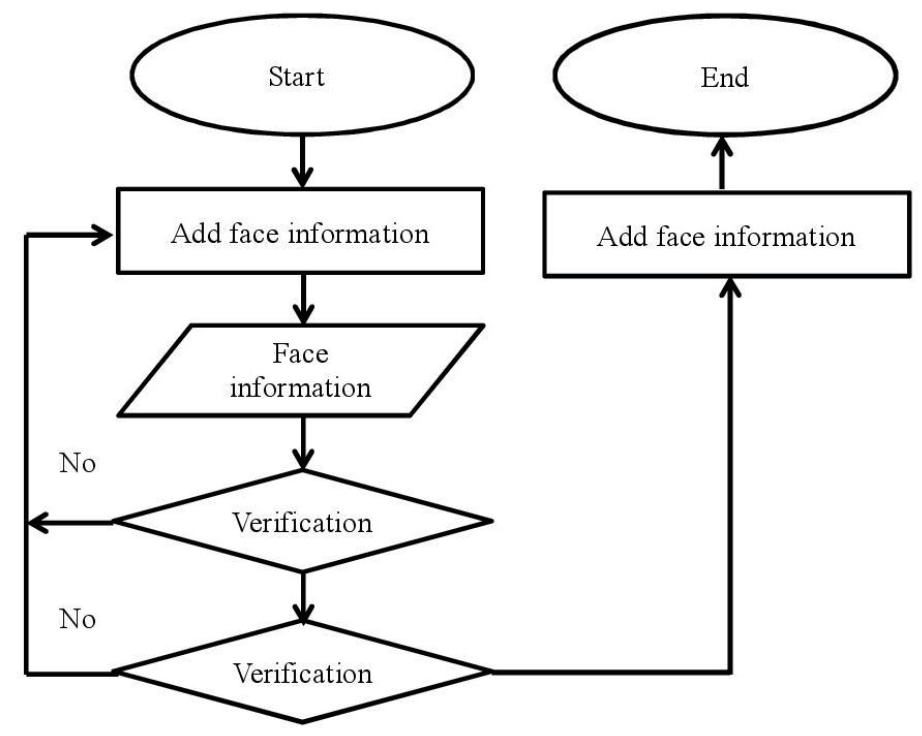

Fig. 1. $\mathrm{B} / \mathrm{S}$ architecture of the system.

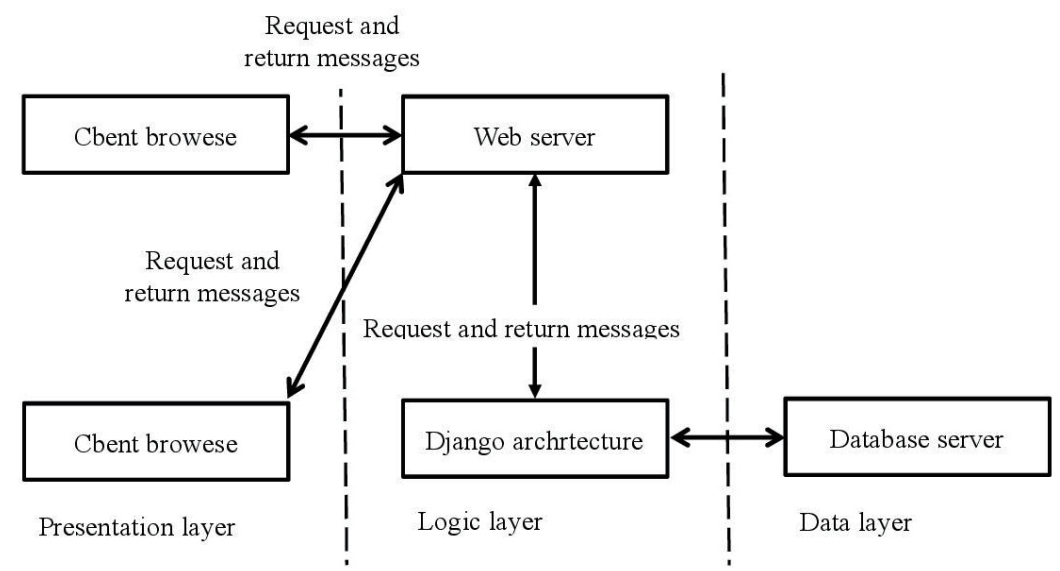

Fig. 2. Flow chart of functional structure of basic face information management.

\subsubsection{Attendance statistics management}

There are two sub-modules in the module managing the employee attendance statistics: one is a module with the function of querying the employee attendance rate. The second is health sensing.

\subsection{System database design}

Database design refers to the construction of an optimized logical model and physical structure for a given application environment, and the establishment of a database and its 
application system based on it, so that it can effectively store and manage data and meet the application needs of various users, including information management requirements and data operation requirements. The corresponding entity relationship (ER) diagram can be derived from entities, their attributes, and associations between entities, as shown in Fig. 3. The database table design uses the ER diagram results for the data structure after the logical design of the database so that the corresponding database tables can be constructed.

\subsection{Database table design}

After the logical design of the database, the ER diagram results obtained above are sorted to construct corresponding database tables, such as the administrator database table shown in Table 2.

\subsection{Detailed modular design of the system}

The table above describes the detailed process of the specific module design of the face recognition attendance system. The detailed design involves the design and compilation of each module or interface of the software system according to the system modular design method, which can be used for reference by developers to write code and form concept diagrams of
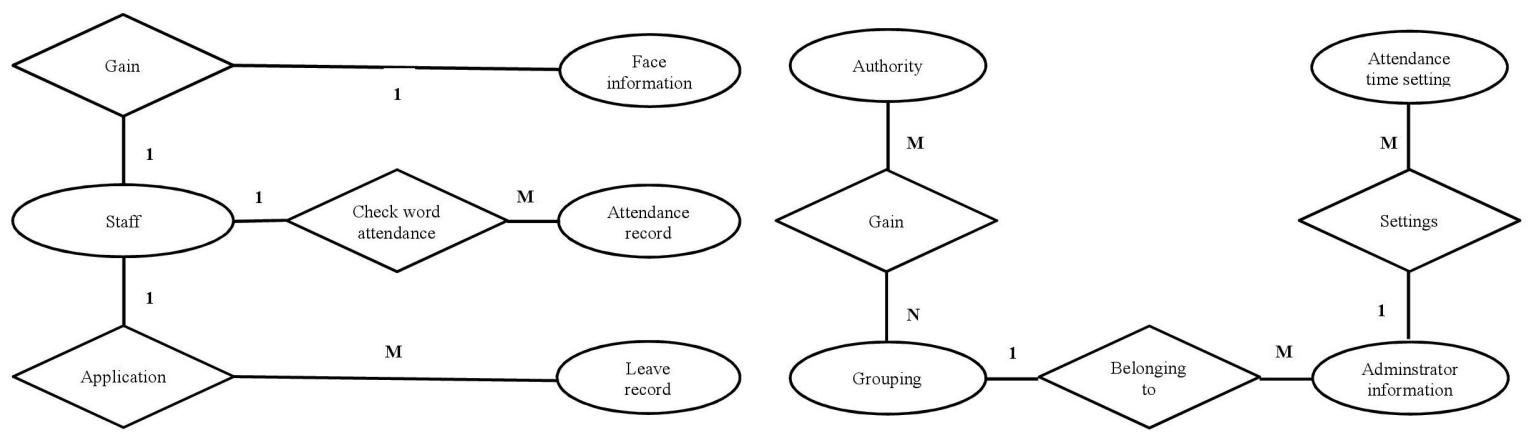

Fig. 3. ER diagram.

Table 2

Administrator database table.

\begin{tabular}{|c|c|c|c|c|}
\hline Table field & Data type & Can it be empty? & Primary key & Remarks \\
\hline id & $\operatorname{int}(11)$ varchar(128) & NOT NULL & PRIMARY KEY & Serial number \\
\hline password & $\operatorname{varchar}(150)$ & NOT NULL & & Password \\
\hline username & varchar(254) tinyint(1) & NOT NULL & & User name \\
\hline email & datetime tinyint(1) & NOT NULL & & Role \\
\hline is_superuser & datetime & NOT NULL & & Mailbox \\
\hline last_login is_staff & & NOT NULL & & Last login time \\
\hline date_joined & & NOT NULL & & State \\
\hline & & NOT NULL & & Joining time \\
\hline
\end{tabular}


modules or component implementation. For example, the flow chart of employee information entry starts when the administrator clicks the employee information management menu, then clicks the "Add" button to enter the information-filling interface, and fills in the relevant information of the employee.

\subsection{System implementation and testing}

After the completion of the system, perform grouping module and functional tests to see if it works. If a bug is found, the problem will be fixed immediately, making the system run properly and reliably. Black box testing is used to detect each function by focusing on the external structure without considering the internal logical structure, while white box testing is used to check wording.

\section{Conclusion and Discussion}

We have discussed the design, operation, and implementation of a system for face recognition, fever sensing, and attendance recording. Because the system has successfully integrated many pieces of software and small hardware, the initial construction cost will be low. It is also energysaving, minimizing maintenance costs. It can also perform fever screenings and track personal health records. The innovations and advantages of this system are listed as follows.

- From the perspective of software engineering project development, the feasibility analysis, requirement analysis, system design and implementation, and system testing are completed.

- The integrated software includes Python, Django, MySQL, HTML, CSS, Java Script, WeChat, face recognition, sensing technology, and an intelligent human body temperature monitoring system.

- The code was written to achieve the front-page layout and uses the H5 interface to open the camera tracking.js to capture the face image, which is combined with a face recognition library to carry out facial recognition comparison. Finally, voice confirmation is used so that the attendance record displays successfully.

- The Django framework and x-admin front-end framework are used to build the background function module, so that employees can view their attendance records through the applet and submit applications for leave.

- The program successfully integrates face recognition, sensing technology, a fever detection attendance system, and the WeChat applet system.

\section{Acknowledgments}

We would like to thank the assisting units of the School of Nursing, Central Taiwan University of Science and Technology, the nurses of the Taichung Blood Donation Center of the Taiwan Blood Foundation, and all the teachers in the Network Engineering Department of the School of Computer Science and Technology of Guangdong University of Petrochemical Technology [Project Number: 2019rc076 (702-519186)]. 


\section{References}

1 Global New Crown Pneumonia (COVID-19) Epidemic Briefing: http://www.medsci.cn/article/show_article. do? id $=025620 \mathrm{e} 215 \mathrm{ed}$ (accessed March 2021).

2 E. Hussein, S. Daoud, H. Alrabaiah, and R. Badawi: Child Youth Serv. Rev. 119 (2020) 105699. https://doi. org/10.1016/j.childyouth.2020.105699

3 R. Kuppuswamy and D. Mhakure: Procedia CIRP 91 (2020) 565. https://doi.org/10.1016/j.procir.2020.02.215

4 R. J. Wotherspoon, C. J. Mannion, and R. E. A. Harlow : Br. J. Oral. Maxillofac. Surg. 58 (2020). https://doi. org/10.1016/j.bjoms.2020.08.114

5 A. Yokoyama: ACM SIGGRAPH 97 Visual Proc. (1997) 114. https://doi.org/10.1145/259081.259204

6 C. Song, M. Ito, Y. Nishiyama, and K. Sezaki: Proc. 3rd ACM SIGSPATIAL Int. Workshop on Prediction of Human Mobility (2019) 33. https://doi.org/10.1145/3356995.3364541

7 L. Mishra, T. Gupta, and A. Shree: Int. J. Educ. Res. Open. 1 (2020) 100012. https://doi.org/10.1016/j. ijedro.2020.100012

8 R. Rameswari, S. Naveen Kumar, M. Abishek Aananth, and C. Deepak: Proc. Mater. Today (2020). https://doi. org/10.1016/j.matpr.2020.04.664

9 S. M. Bah and F. Ming: Array 5 (2020) 100014. https://doi.org/10.1016/j.array.2019.100014

10 K. B. Pranav and J. Manikandan: Procedia Comput. Sci. 171 (2020) 1651. https://doi.org/10.1016/j. procs.2020.04.177

11 D. J. Lu, M. Girgis, J. M. David, E. M. Chung, K. M. Atkins, and M. Kamrava: Adv. Radiat. Oncol. 9 (2020) 100576. https://doi.org/10.1016/j.adro.2020.09.016

12 M. Katarahweire, E. Bainomugisha, and K. A. Mughal: Dev. Eng. 5 (2020) 100054. https://doi.org/10.1016/j. deveng.2020.100054

13 D. Sethia, D. Gupta, and H. Saran: Smart Health 13 (2019) 100063. https://doi.org/10.1016/j.smh1.2018.11.001.

14 M. L. Challa and K. L. S. Soujanya: Proc. Mater. Today 37 (2020) 2109. https://doi.org/10.1016/j. matpr.2020.07.536

15 F. Liu, G. Liu, Q. Zhao, and L. Shen: Neurocomputing 402 (2020) 14. https://doi.org/10.1016/j. neucom.2020.03.102

16 D. K. Jain, X. Lan, and R. Manikandan: Image Vis. Comput. 103 (2020) 104024. https://doi.org/10.1016/j. imavis.2020.104024

17 Q. Hu, S. Yin, H. Ni, and Y. Huang: Procedia Comput. Sci. 174 (2020) 505. https://doi.org/10.1016/j. procs.2020.06.118

18 U. Jayaraman, P. Gupta, S. Gupta, G. Arora, and K. Tiwari: Neurocomputing 408 (2020) 231. https://doi. org/10.1016/j.neucom.2019.08.110

19 M. Kim, S. Kim, and J. Kim: Telemat. Inform. 38 (2019) 46. https://doi.org/10.1016/j.tele.2019.02.003

\section{About the Authors}

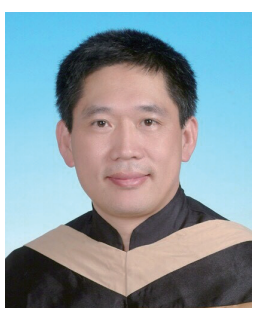

Min-Chuan Huang received his bachelor's degree from the Department of Mechanics, Taiwan Military Academy, in 1984, and his M.S. degree in science and Ph.D. degree in engineering in 2003 and 2011, respectively, from I-Shou University. He has been an associate professor at Guangdong Institute of Petrochemical Industry since September 2019. His main research interests are information engineering, big data, deep learning, investment, sociology, and game education. (h0912259520@gmail.com )

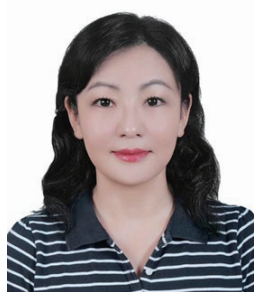

I-Ping Chen received her bachelor's degree in nursing from Central Taiwan University of Science and Technology in 2004. Since 2019, she has been studying for a master's degree at the Nursing Institute of the School of Nursing, Central Taiwan University of Science and Technology. Her main research interests are elderly care, blood donation education, platelet donation promotion, chronic disease care, and hospice nursing education.

(iping.tc@gmail.com ) 


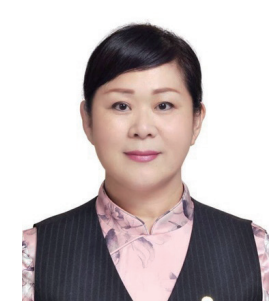

Hsiang-Lin Huang obtained a bachelor's degree from Xingwu University of Science and Technology in 2008 and an M.S. degree from the School of Management at Mingchuan University in 2012. She has been a university lecturer since 2013. Her main research interests are Chinese language teaching verification, environmental education, cultural heritage preservation, real estate investment, and homestay management.

(rose.kinmen@gmail.com )

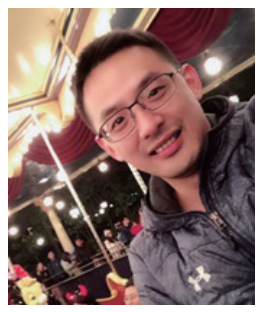

Shih-Fu Sung obtained his Ph.D. degree in information engineering from I-Shou University, Taiwan, in 2016 and is currently an assistant professor at I-Shou University. His areas of study are information management, big data, IoT application, marketing management, and business management. f(poem5203@gmail.com )

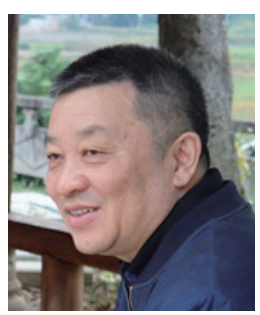

Ai-Guo Wang obtained his bachelor's degree from Jilin Institute of Technology in 1989. Since 2015, he has been an associate professor and director of the Network Engineering Department of Guangdong University of Petroleum Technology. His main research interests are network engineering, computer software, and programming. (jlwag@126.com ) 